\title{
Article \\ Understanding the Emotional Intelligence Discourse on Social Media: Insights from the Analysis of Twitter
}

\author{
Shardul Shankar * and Vijayshri Tewari
}

Citation: Shankar, Shardul, and Vijayshri Tewari. 2021. Understanding the Emotional Intelligence Discourse on Social Media: Insights from the Analysis of Twitter. Journal of Intelligence 9: 56. https://doi.org/ 10.3390/jintelligence 9040056

Received: 23 September 2021 Accepted: 11 November 2021 Published: 24 November 2021

Publisher's Note: MDPI stays neutral with regard to jurisdictional claims in published maps and institutional affiliations.

Copyright: (c) 2021 by the authors. Licensee MDPI, Basel, Switzerland. This article is an open access article distributed under the terms and conditions of the Creative Commons Attribution (CC BY) license (https:// creativecommons.org/licenses/by/ $4.0 /)$.
Department of Management Studies, Indian Institute of Information Technology, Allahabad 211015, India; vijayshri@iiita.ac.in

* Correspondence: imp2016001@iiita.ac.in

\begin{abstract}
Social networks have created an information diffusion corpus that provides users with an environment where they can express their views, form a community, and discuss topics of similar or dissimilar interests. Even though there has been an increasingly rising demand for conducting an emotional analysis of the users on social media platforms, the field of emotional intelligence (EI) has been rather slow in exploiting the enormous potential that social media can play in the research and practice of the framework. This study, thus, tried to examine the role that the microblogging platform Twitter plays in enhancing the understanding of the EI community by building on the Twitter Analytics framework of Natural Language Processing to further develop the insights of EI research and practice. An analysis was conducted on 53,361 tweets extracted using the hashtag emotional intelligence through descriptive analytics (DA), content analytics (CA), and network analytics (NA). The findings indicated that emotional intelligence tweets are used mostly by speakers, psychologists (or other medical professionals), and business organizations, among others. They use it for information dissemination, communication with stakeholders, and hiring. These tweets carry strong positive sentiments and sparse connectedness. The findings present insights into the use of social media for understanding emotional intelligence.
\end{abstract}

Keywords: emotional intelligence; Twitter; social media; emotional analysis; sentiment analysis; network analytics

\section{Introduction}

Of late, cyberspace and social media have become increasingly adjacently parallel to the physical world, especially when it comes to conversations or discourse (Wei et al. 2019). Social networks have allowed for easier connections with people right from a person's couch and they have increased the convenience of people's daily lives, but they have also opened people's minds regarding security and privacy concerns that come with the ease of use of these platforms. The industry-academic community has grown significantly interested in studying the potential use that these social networks' data could provide to businesses or the research community. The large quantities of data generated (commonly called "big data") allow researchers to extract information from these data, create new insights into the different domains, and understand users' characteristics, behavior, and decision-making patterns. Literature regarding social media data has studied the demographical characteristics of the users (Jurgens et al. 2015), the users' psychological traits expressed through social networks (Burrus et al. 2012; Chen et al. 2017; Kosinski et al. 2014; Xu et al. 2008), stock price predictions (Huang and Liu 2020; Tsui 2017), epidemics and pandemics (Cinelli et al. 2020; Zhao et al. 2020; Kadam and Atre 2020; Gao et al. 2020), elections (Jaidka et al. 2019; Ceron et al. 2016; Ferrara 2020), brand management (Jin 2012; So et al. 2018), information diffusion (Stieglitz and Dang-Xuan 2013; Kushwaha et al. 2020), public opinion (Gorodnichenko et al. 2018; Ford et al. 2019; Hickerson and Kothari 2017), and healthcare (Terry 2009; Courtney et al. 2013; Pizzuti et al. 2020). 
The domain of emotional intelligence (EI) has been relatively dawdling when it comes to utilizing the potential that social network platforms and their data can play in predicting the users' emotional intelligence. Even though there has been growth in recent times in using social media to understand the facets of EI (Menon and Rahulnath 2016; Hornung et al. 2018; Madaan et al. 2020), these studies have relied on traditional methods of data collection and self-reporting techniques (e.g., survey techniques or interviews) and statistical techniques (e.g., moderation and mediation analyses). Nevertheless, there has been a recent shift in using big data in the area of EI (Wei et al. 2019; Abkenar et al. 2020). Even though organizations are perfectly capable of generating big data on their employees, social media platforms (e.g., Facebook, Twitter, YouTube, Reddit, etc.) make the vast majority of contributions towards these big data.

A study (Cecere 2012) encompassing leaders and industry heads found out that onethird of modern organizations are using big data to understand their employees. The organizations also believed that the traditional means of generating big data are competent for effectively managing behavioral and transactional data, but were uncertain about using social media data for generating business intelligence. This was echoed in other industry surveys (Natoli 2013; Mann 2014), where business decisions were being effectively leveraged by these organizations, but less than $1 \%$ explained that they were able to use social media for human resource planning and organizational behavior. They believed that social media would transform organizational psychology by effectively studying the emotional intelligence of their employees, but a majority of these organizations had no idea where to even start looking for it.

In our study, we concentrated our efforts on one particular social media giant, Twitter (Twitter 2021). This specific social media platform was chosen over its competitors because of its microblogging capability and the fact that it is arguably the fastest growing social media platform there is (Sharma et al. 2017). Twitter is increasingly used by its users for multiple reasons, including discussing mental issues, sharing news and personal feelings, or expressing opinions about political and ideological themes in a brand/organization/celebrity discussion (Chae 2015). The industrial community or researchers can access Twitter data through their APIs (application programming interface) to analyze the data for various domains of study.

This study was carried out to build upon the understanding of social media in the context of emotional intelligence. This is achieved by using natural language processing techniques to analyze tweets containing contexts of emotional intelligence, and the associated people or users who were discussing EI on the Twitter platform, to develop insights into EI practices and research and the potential role that Twitter can play in this. This thought was echoed in studies on industry experts and organizations (Chae 2015; Cecere 2012; Zhang et al. 2019), as there is a clear lack of an insightful understanding of the concept, and there is very little literature to support this understanding.

Even though there have been a lot of proposed frameworks for understanding emotion expression, textual emotion, and the underpinnings of emotions in real-time data, there is a significant lack in the literature when it comes to creating a framework that understands the contextual understandings of the discourse of emotional intelligence, especially in social media (Israelashvili et al. 2021). The motivation of this study was to propose a framework that can be used to better understand how emotional intelligence is discussed in social media and social networks, and how these discussions are driving understandings of the emotional psyche. This study would thus try to create a framework on which future researchers and industries can build to generate knowledge from Twitter data in the EI community.

Chae (2015) described Twitter Analytics (TA) as an analytical technique for analyzing Twitter data for a research outcome. They stated that TA is a combination of three analytics-descriptive analytics (DA), content analytics (CA), and network analytics (NA). We have tried to modify this framework to extract the information pertaining to emotional intelligence. These three analytical techniques focus on multiple magnitudes of Twitter 
data. The collected tweets and metadata covered the discussions of individual users, professionals, and organizations in terms of the concept of EI. Specifically, the findings of the analyses have tried to answer these research questions:

(1) Are there any patterns in the characteristics of the information diffusion of emotional intelligence tweets?

(2) Are there any dominant topics, content, or discussions that are being shared on Twitter regarding emotional intelligence?

(3) Are there any patterns in the characteristics of the Twitter users who indulged in dialogues on emotional intelligence?

(4) Are there any patterns in the sentiments of the emotional intelligence tweets, and what are the tweet contents that contain sentiments of emotional intelligence?

Accordingly, the research is divided into sections as follows: Section 2 presents the present literature about the use of Twitter in multi-dimensional domains, Section 3 presents the data collection and pre-processing methodologies, Section 4 discusses the framework of Twitter Analytics, Section 5 provides an analysis of the collected tweets using TA, and the final sections conclude the study by presenting the research implications, limitations, and scope for future research.

\section{Literature Review}

Since its inception in 2006, Twitter has become one of the biggest microblogging websites, with 500 million daily and 200 billion yearly tweets (Twitter 2020), and 150 million monetizable users (Tankovska 2021). A study found out that over $75 \%$ of Fortune 500 companies have an active Twitter account, for their corporates and their brands (Malhotra et al. 2012). It has become one of the fastest information dissemination tools that allow for discussions, conversations, and even the spread of information that is true or false, making it one of the strongest assets for anyone with a voice.

A tweet (Vega et al. 2010), which is Twitter's shared content, contains 280 characters, through which the users share their opinions and have real-time conversations. A tweet can be one of three types: an original tweet, a retweet, or a reply (Chae 2015). All of these messages can be traced manually or by using Twitter's application programming interface (API). A popular tweet usually gets a status of "trending", which helps for easier reach and conversations with followers (Aiello et al. 2013).

Due to its increasing popularity, Twitter is being used in varied domains of practical and academic research, including stock market forecasting and predictions (Bollen et al. 2011; Sóti et al. 2020), brand management (Zimbra et al. 2016; Lalicic et al. 2020), crisis management (Kersten and Klan 2020; Wang et al. 2021), healthcare (Alotaibi et al. 2020; Masip et al. 2020; Talbot et al. 2021), finance (Souza et al. 2015; Mao et al. 2011), information technology and information systems (Castillo et al. 2011; Ruz et al. 2020), supply chain management (Chae 2015; O'leary 2011), and psychology (Bogen et al. 2020; Dodds et al. 2011).

When talking about emotions and emotional intelligence, the domains of psychology, philosophy, sociology, organizational behavior, etc. have been extensively researched over a long time (Sailunaz and Alhajj 2019; Bryan and Mayer 2021). Initially, it was a part of biological evaluation, but with time, neuroscience has opened avenues for evaluating emotions as a socio-cognitive function (Tago and Jin 2018).

This shift in understanding the influence that emotional intelligence has on the limbic as well as neo-cortex systems, thereby creating a function of the neural system, has allowed the extraction mechanisms to become diverse-from primary and secondary data to more comprehensive experimental, experiential, and real-time big data and natural language processing (Tellez et al. 2017; Israelashvili et al. 2021).

Many researchers have proposed their own frameworks for evaluating textual emotions (Binali et al. 2010; Jain et al. 2017) but these frameworks have a high presence of linguistic and methodological limitations (Sailunaz and Alhajj 2019). Moreover, these 
studies have focused primarily on understanding emotional triggers and have skimmed over the parameters of emotional regulation and intelligence.

This study also fights the criticism of previous studies (Hall and Mast 2007) that the verbal components of emotional discourse are usually missing in the evaluation of emotional intelligence. To address these issues, there have been several studies that have used real-time data to understand the underpinnings of the concept of emotion (Paul and Sui 2019; Suhasini and Srinivasu 2020; Lim and Birney 2021; İŞ and Tuncer 2018).

Yet, despite the recent increase in the interest in using Twitter as a platform to study the domain of emotions, studies in the area of emotional intelligence are very scarce. One exception (Kumar and Devi 2020) used EI to study the perceptions of political parties. The objective of the present study was to identify the relationship between the tweet contents of emotional intelligence tweets among users, professionals, and organizations. The findings would contribute to the impact that Twitter has when it comes to understanding emotional intelligence in life and the workplace.

\section{Data}

The data collected for the proposed framework required the effort of manual classification of the tweets for the analysis of the extracted tweets. The initial extraction was performed using keywords such as "emotional intelligence", "ei", "eq" etc. and this gave us the understanding that \#emotionalintelligence was the most prevalent hashtag that could be used in the study. The tweets were collected from 14th February 2021 to 6th March 2021, and which included 53,361 emotional intelligence tweets and their content.

The study was conducted using Python (version 3.8.7) using the Twitter API and the tweepy package of Python via Twitter. The tweets collected were public tweets, and private tweets were excluded from the collection (Gokulakrishnan et al. 2012). The privacy of the users was also maintained in the process, as the personal or private information of the users was removed from the analysis. A summary of the dataset is shown in Table 1.

Table 1. Summary of the dataset.

\begin{tabular}{lc}
\hline Dates of Data Collection & 14 February 2021-6 March 2021 \\
\hline Unique Users & 22,895 \\
\hline No. of Tweets Collected & 53,361 \\
\hline Maximum Number of Retweets on a Tweet & 193 \\
\hline Maximum Number of Favourites & 1801 \\
\hline Day with the Maximum Tweets & Monday \\
\hline Hour with the Maximum Tweets & $20: 00$ \\
\hline Date with the Maximum Account Creation by Users in the Data & 25 February 2016 \\
\hline
\end{tabular}

\section{Data Pre-Processing}

The tweets of any user at any given time are usually of three types: textual tweets, visual tweets, and auditory tweets. To make the information in them useful, a great amount of data cleaning was required, also known as data pre-processing. This was achieved by creating data tokens and using only the textual tweets, according to the process explained in Angiani et al. (2016) and Gokulakrishnan et al. (2012). The steps involved in data pre-processing were:

- Fixing grammatical, spelling, and punctuation errors;

- Fixing slang, acronyms, and colloquialisms;

- Removing numbers and digits;

- Removing exercising, gym, and workout data;

- Removing URLs by searching for http/https/www and removing the following text;

- Removing contractions and negations;

- Removing emojis and emoticons; 
- $\quad$ Removing non-ASCII characters (including non-UTF-8 Unicode);

- Removing stop-words and extra spaces;

- Converting all the text to lower case;

- Stemming and lemmatizing the words

\section{Framework for Twitter Text Analytics}

While data collections from social media platforms such as Twitter rely on APIs, the analysis is usually challenging, as the data have a lot of noise, are unstructured, and are substantially enlarged and enriched (Doldor et al. 2019) in comparison with their traditional counterparts. An analytical framework is also not readily available (Zeng et al. 2010), and hence, a framework encompassing the methods that extract and evaluate information from the data is required. The framework used in this study was initially developed in a study by Chae (2015) to analyze the Twitter dataset of supply chain tweets, which has been modified to evaluate the discourse on emotional intelligence. This framework has three analytics: descriptive analytics, content analytics, and network analytics. Figure 1 presents the relevant metrics of the analytical processes.

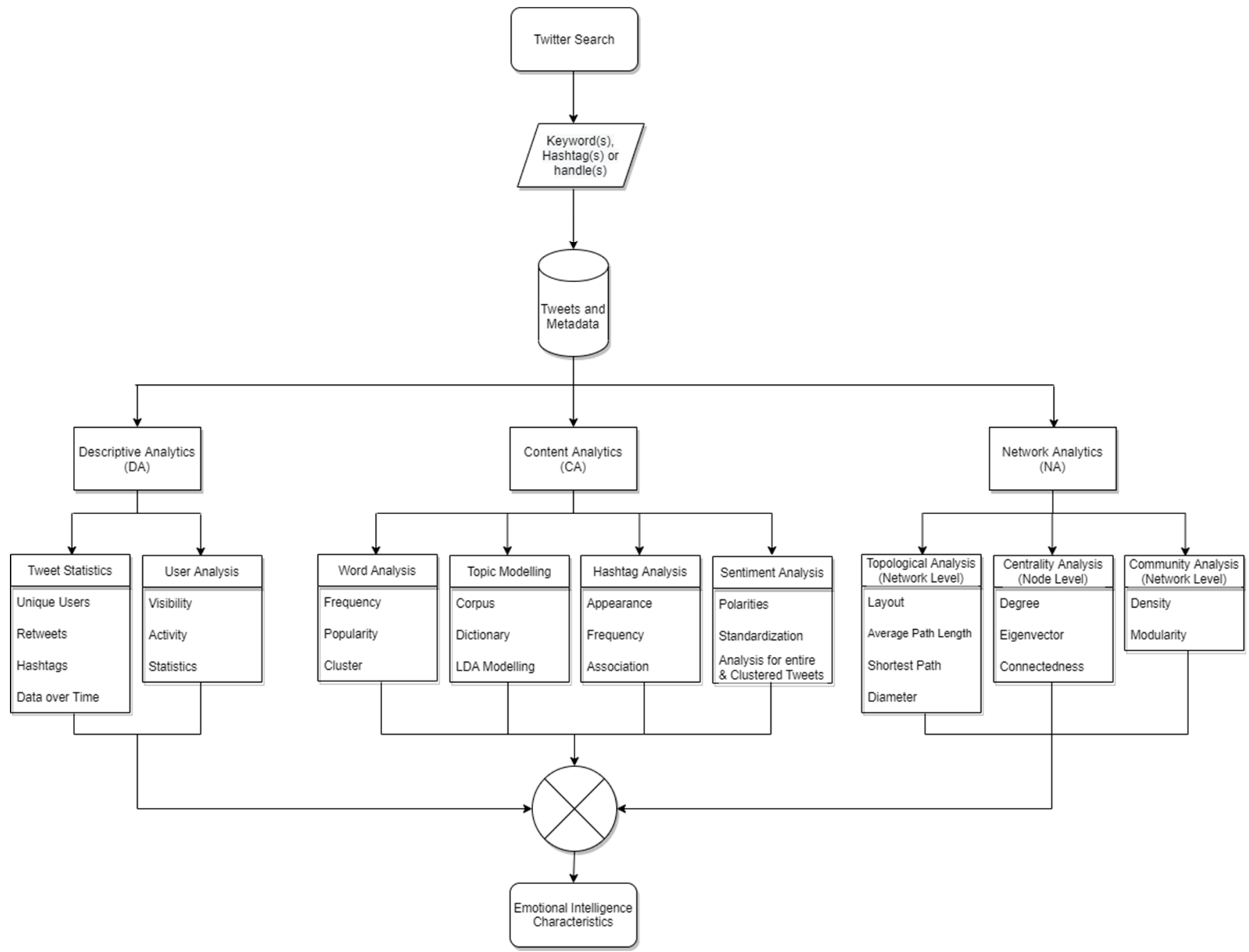

Figure 1. Research process framework for Twitter Analytics. 


\subsection{Descriptive Analytics (DA)}

In this process, we focused mainly on the descriptive statistics of the dataset. The descriptive metrics and the user metrics gave us direction into other user-related information that was used in the content and network analytics.

\subsection{Content Analytics (CA)}

The data collected were primarily unstructured in nature, and hence, natural language processing (NLP) was used to pre-process, format, and transform the data for word analysis, topic modeling, and sentiment analysis.

\subsection{Network Analytics (NA)}

With the help of the data and text obtained through the above processes, a network model was created using the GUI tool Gephi (Bastian et al. 2009). The nodes were the Twitter users and the edges were the relationships between these users.

\section{Results}

\subsection{Descriptive Analytics}

Descriptive analytics of the data from social media platforms is the initial building block for analyzing the social media data, not only for businesses but also researchers. DA was performed using the Python package Gensim (Cao et al. 2009) by using the understandings developed by Bruns and Burgess (2011), along with other statistical techniques. With the help of Python and its package, information about the users and tweets were extracted, and statistical techniques were used to visualize the statistics of the data.

\subsubsection{Tweet Statistics}

Out of 53,361 tweets, unique users accounted for $41.36 \%(22,895)$, retweets accounted for $28.29 \%(15,096)$, and unique mentions accounted for $7.33 \%(4056)$, respectively. In total, 11,910 hashtags were used in these tweets, covering the four important factors of a traditional emotional intelligence model (motivation, self-awareness, empathy, etc.).

\subsubsection{User Analysis}

There were 22,895 unique users in the dataset, indicating that every user, on average, sent 2.33 tweets, 1.52 retweets, and 0.81 mentions. Active users were calculated by using the formula (tweets + retweets + mentions), and visible users by the formula (retweets + mentions received). Figure 2 shows the active users and the visible users. The figure shows that the most active users were also the most visible users, which was expected. One important finding was that amongst the most active and visible users were the speakers that talk about motivation, emotional intelligence, and other soft skill topics.

\subsection{Content Analytics}

\subsubsection{Word Analysis}

The most popular words found in the tweets were motivation (49,530 times in tweet texts), inspiration (13,383 times), empathy (2872 times), self-belief (1740 times), self-love (1566 times), care (1325 times), inspire (1328 times), emotional intelligence (1200 times), and self-care (1077 times), among others.

\subsubsection{Topic Modeling}

To further classify the clustering of the words, we used the topic modeling technique using Python's Gensim package by creating a corpus and dictionary, according to the algorithm of Cao et al. (2009), and used these as inputs in LDA modeling. We were able to create four distinct topics with eight words in each topic. Table 2 presents the topic modeling outcomes from the word analysis. 


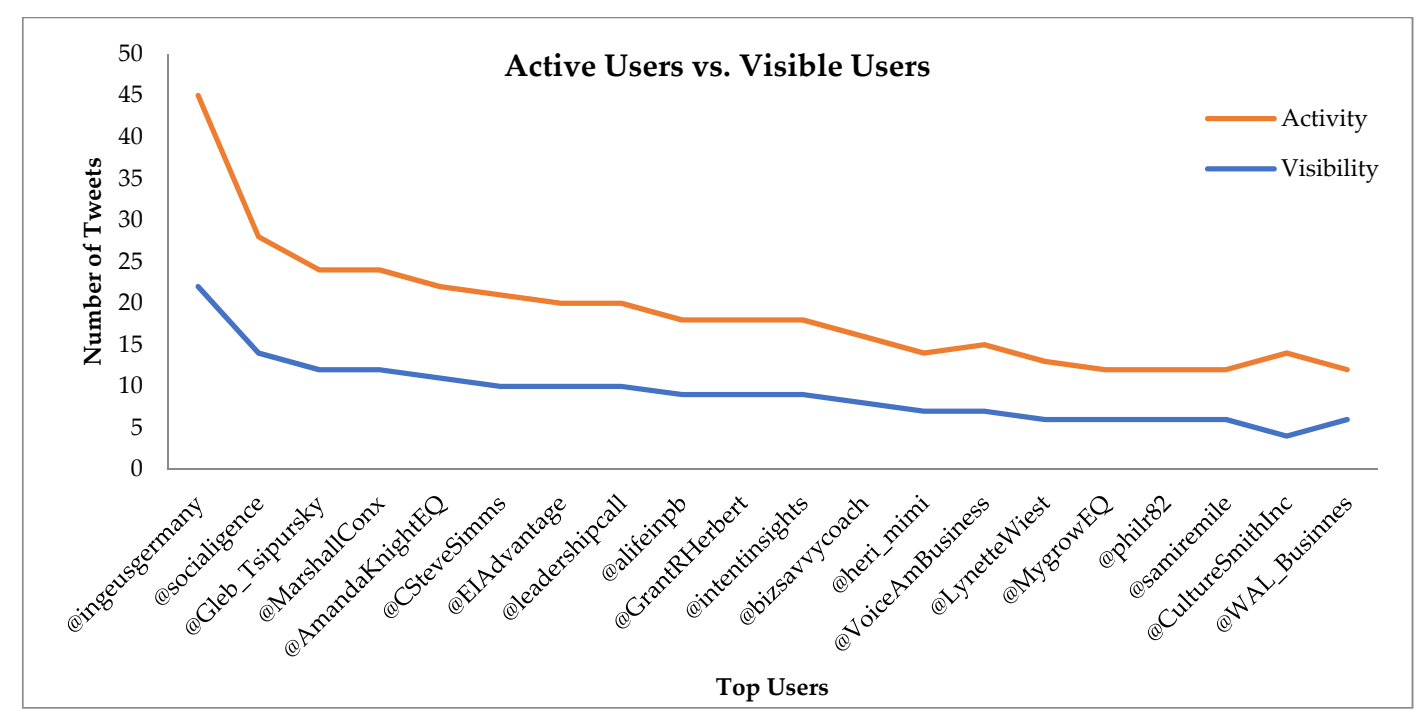

Figure 2. Most active vs. visible users.

Table 2. Topic modeling and word analysis of the corpus.

\begin{tabular}{|c|c|c|c|c|c|c|c|}
\hline Emotional Intelligence & Frequency & Self-Awareness & Frequency & Empathy & Frequency & Motivation & Frequency \\
\hline emotional intelligence & 1200 & self-belief & 1740 & empathy & 2872 & motivation & 49,530 \\
\hline eq & 546 & self-love & 1566 & care & 1325 & inspiration & 13,383 \\
\hline emotions & 368 & well-being & 398 & self-care & 1077 & inspire & 1328 \\
\hline intelligence & 293 & awareness & 289 & compassion & 426 & motivationalthoughts & 1318 \\
\hline emotional health & 67 & self-awareness & 232 & understanding & 178 & determination & 406 \\
\hline emotional quotient & 48 & belief & 142 & insight & 140 & inspired & 280 \\
\hline ei & 41 & mental health & 97 & compassionate & 41 & commitment & 95 \\
\hline emotionalwellbeing & 22 & workplacewellbeing & 33 & self-compassion & 40 & self-motivation & 70 \\
\hline
\end{tabular}

\subsubsection{Hashtag Analysis}

In total, 45,859 hashtags were found in the tweets, with the total occurrence of these hashtags being 447,747 times. The most popular hashtags were \#motivation, \#inspiration, \#emotionalintelligence, \#success, \#goals, \#empathy, \#positivity, \#happiness, \#mindfulness, \#selflove, \#wisdom, \#believe, \#training, and \#selfcare, including others. This showed that there were, on average, 8.39 hashtags per tweet, and the top three hashtags accounted for $15.74 \%$ (70,476 times) of the overall hashtag appearance in the tweets.

\subsubsection{Sentiment Analysis}

Sentiment analysis was mostly done using the Python package Textblob (Zhang et al. 2018), and the tweets were categorized into three major polarities, i.e., positive, negative, and neutral. Table 3 presents the percentages of the polarities from the sentiment analysis of our tweet data. The overwhelming majority of emotions of these tweets were of positive sentiment, with the smallest percentage for positive sentiment being self-awareness, with $88.53 \%$. Neutral sentiments were also higher than negative sentiments in our topics, indicating that when the sentiments were not positive, they were tending towards neutral sentiments. Negative sentiments regarding the topics were very meager, with the largest percentage being $2.95 \%$, attributed to self-awareness.

To visually understand the sentiment analysis of the tweet, the first step was to create a tabulated representation of the percentage sentiment analysis of the tweet topics. Figure 3 shows a graphical representation of the percentage sentiment analysis of the tweet topics. 
Table 3. Sentiment analysis of the tweet topics (in percentages).

\begin{tabular}{cccc}
\hline Topics & Negative & Neutral & Positive \\
\hline Emotional Intelligence & $2.61 \%$ & $4.76 \%$ & $92.63 \%$ \\
Self-Awareness & $2.95 \%$ & $8.51 \%$ & $88.53 \%$ \\
Empathy & $2.16 \%$ & $4.37 \%$ & $93.47 \%$ \\
Motivation & $1.22 \%$ & $4.16 \%$ & $94.61 \%$ \\
\hline
\end{tabular}

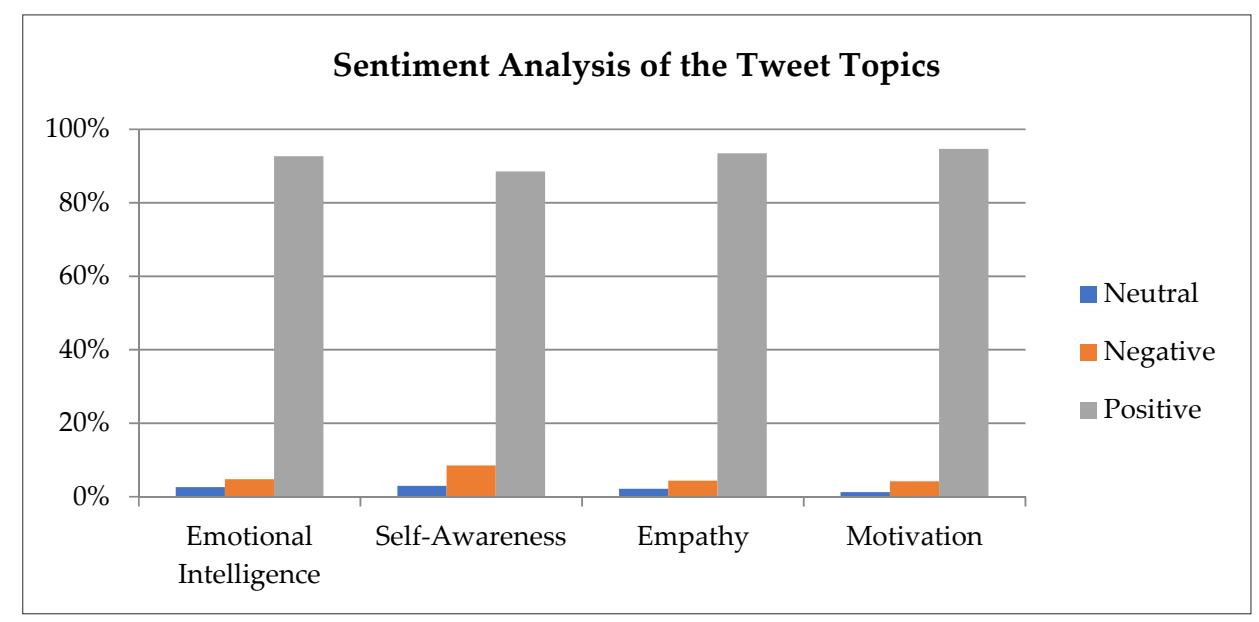

Figure 3. Tabular representation of the sentiment analysis of the tweet topics (in percentages).

Next, word clouds of the positive and negative sentiments were created. This was also achieved using the Textblob package of Python. Figure 4 shows the word clouds of the two sentiments according to their frequencies.

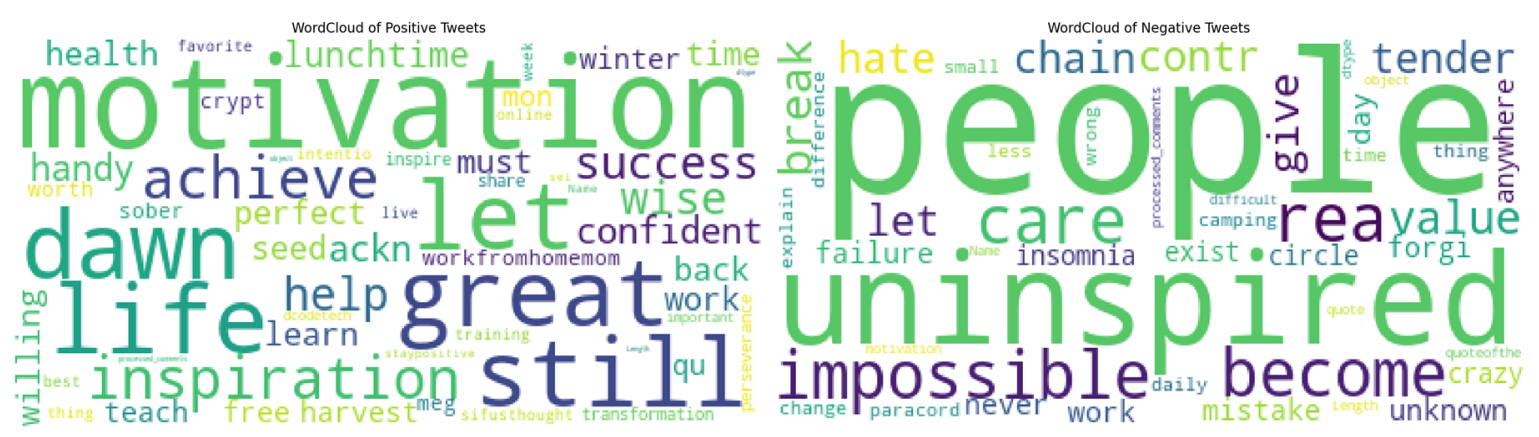

Figure 4. Word cloud of the sentiments of tweet topics.

It can be seen that motivation, great, life, and dawn were the most frequently occurring positive words, and people, uninspired, impossible, and insomnia were the most frequently occurring negative words.

Standardization of these sentiments $[-1,0,1]$ was achieved using the SentiStrength package of Python (Thelwall et al. 2011). Even after standardizing the sentiment polarities of the topics, the normal distribution of these sentiments was pushed towards the positive side of the distribution. Figure 5 shows the normal distribution of the sentiment polarities of the dataset.

It can be seen from the figure that even though the distribution of sentiment polarity is normally distributed, most of the values of the curve are in the positive region of the curve.

A similar finding was observed when the entire dataset of the analysis was tested for sentiment analysis without the topics. Figure 6 shows the sentiment analysis of the entire dataset at the non-topical level. There was an obvious skew of the sentiment 
polarities towards the positive sentiment, with $68.44 \%$ of all the tweets ranging from 0 to +1 , indicating an overall positive sentiment within the dataset.

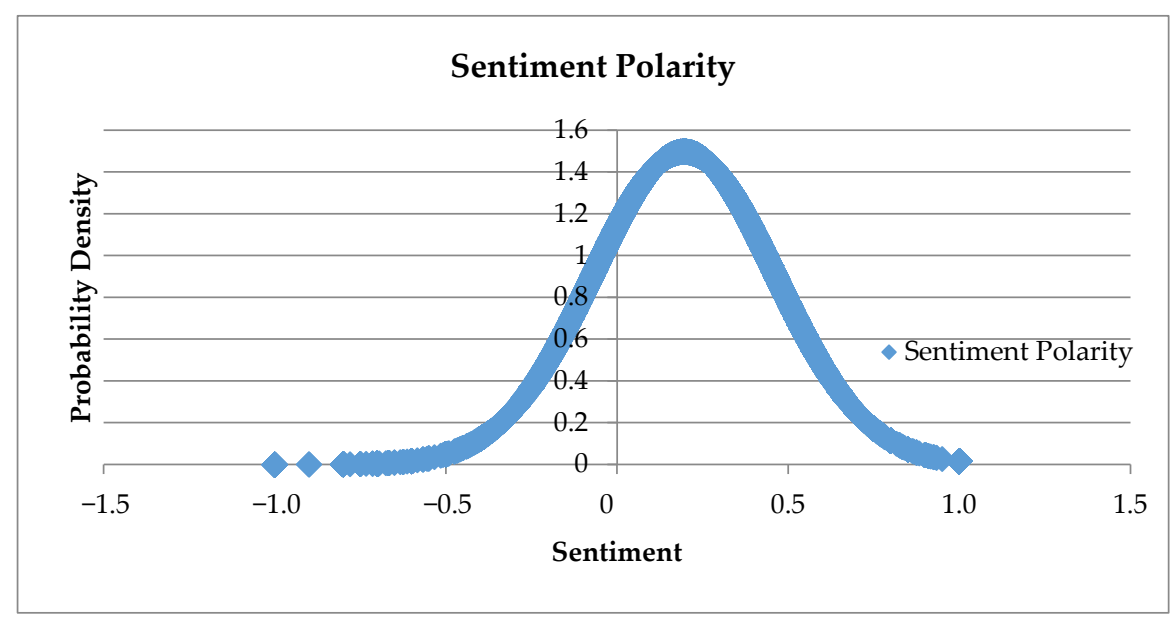

Figure 5. Standard normal distribution of the sentiment polarities of the topics.

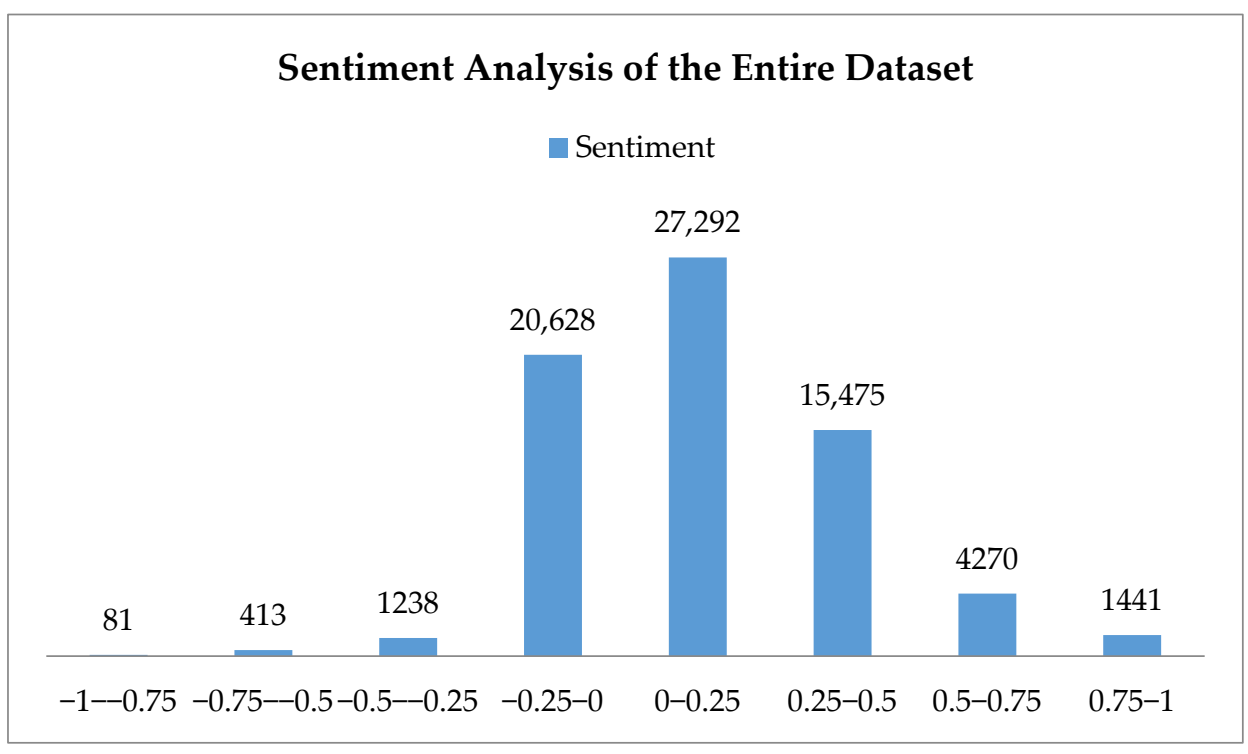

Figure 6. Sentiment analysis at the dataset level of the entire dataset.

\subsection{Network Analytics}

A topological social network was created to understand the node and network-level metrics of the user data. The network graph was created using the open-source software known as Gephi (Bastian et al. 2009). The nodes of the graph were the users that had interactions with each other using emotional intelligence as a content topic, and the edges were the mentions that the users gave or received using the @mentions process (Chae 2015). There were 4058 nodes and 217 edges. The network diameter was 5, indicating that the network was tight but sparse. Figure 7 shows the network graph of the distribution. The average path length was 2.405, which indicates that all the nodes were, on average, at least three nodes away from each other. This is consistent with the typologies of other networks studied in the domains of emotions (Watts 2004; Moeller et al. 2018). 


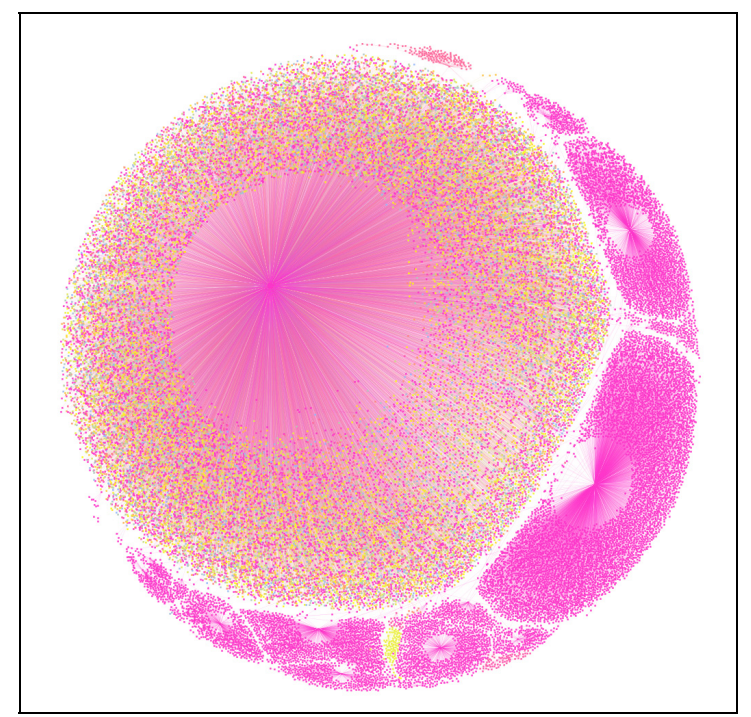

Figure 7. Network diagram of "emotional intelligence" in tweet data.

To figure out the node connectedness, the in-degree of all the nodes was calculated to figure out the nodes' popularity. The findings showed that speakers (@DriverClassics, @gvhawtin), doctors (@Gleb_Tsipursky, @denisemose), and businesses (@i_GotQ, @MotivationBytes) were amongst the most popular, with high tweets and mentions. Table 4 presents a tabulation of high in-degree users.

Table 4. List of high in-degree users.

\begin{tabular}{|c|c|c|c|}
\hline Label & In-Degree & Out-Degree & Degree \\
\hline iGotQ & 24 & 19 & 43 \\
\hline Jayson Waller & 19 & 17 & 36 \\
\hline Allan Beveridge & 17 & 13 & 30 \\
\hline Dr. Gleb Tsipursky & 15 & 11 & 26 \\
\hline Motivational Bytes & 14 & 10 & 24 \\
\hline Waritha & 12 & 12 & 24 \\
\hline 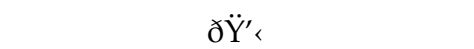 & 10 & 5 & 15 \\
\hline Thomas & 9 & 10 & 19 \\
\hline Gemma Hawtin $ð \ddot{Y}^{\prime т м}$ & 8 & 10 & 18 \\
\hline 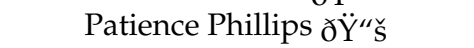 & 7 & 15 & 22 \\
\hline$\hat{a} \sim i_{s}$ keep pushing the limits $\hat{a} \sim i_{s}$ & 7 & 9 & 16 \\
\hline The Obsidian Dragon & 7 & 1 & 8 \\
\hline PeopleSkillsChat & 4 & 3 & 7 \\
\hline STEERus & 4 & 3 & 7 \\
\hline Money Making Conversations & 4 & 8 & 12 \\
\hline Joaquin Salamanca & 4 & 5 & 9 \\
\hline Super-A & 4 & 7 & 11 \\
\hline Nikki Chopra & 3 & 1 & 4 \\
\hline Debesh Choudhury & 3 & 4 & 7 \\
\hline EQpassion & 3 & 2 & 5 \\
\hline
\end{tabular}

The graph also showed that the connectedness had 5\% representation on average, further explaining that the network is sparse, with the largest community having $6 \%$ of total user representation, with the smallest community having $4 \%$ of user representation.

\section{Discussion}

The study was conducted on 53,361 tweets to examine the characteristics of the emotional intelligence tweets and the users discussing the topic. In the preliminary secondary analysis of the topic, it was clear that the literature in this domain is somewhat scarce, as 
multiple articles have discussed emotions in Twitter users and their tweets (Wang and Pal 2015; Dale et al. 2020), but there is relatively negligible research covering emotional intelligence in the Twitter users (Zhang et al. 2019). This study was thus carried out to create a branch of findings that can be used to add to the existing literature on the study of emotional intelligence.

The rate at which people talk about emotional intelligence on Twitter was contrary to what was expected, as across the data collection timeframe, the discussions were fairly consistent, with almost similar numbers of users, tweets, replies, or mentions being shared and discussed (see Table 1), but the interesting finding was the rate at which hashtags were used in those tweets. Even though a previous study found that users, especially professionals, used 12-23\% of hashtags in their tweets (Bougie et al. 2011), our study found that $85.94 \%$ of users used hashtags, with two or more hashtags in their tweets, which is consistent with previous findings (Chae 2015). An average of five hashtags was used in the tweets, and a surprisingly high number for \#motivation was found.

One of the interesting findings of this study was that many popular tweets were shared by people in the motivational speaking business, closely followed by people working in the psychological profession, and employees. Nearly all of the tweets were originally created tweets. It was also apparent that the most active users were the most visible users as well. This also showed that the majority of tweets were made by a very small percentage of users, which was backed by the centrality analysis of the network diagram. The first $9 \%$ of the users accounted for $56 \%$ of the tweets, which is consistent with previous studies (Zafar et al. 2020).

In analyzing the sentiments of the tweets, the findings suggested that the overwhelming majority of the emotions were positive (as shown in Figure 4), which was consistent after standardizing the values of the sentiments. Good topic modeling performance was achieved in the study using the LDA model, which provided us with four distinct topics on which topic modeling could be carried out and the sentiment analysis could be modeled around. The four topics found were emotional intelligence, self-awareness, empathy, and motivation. The highest positive sentiment was found for motivation and the lowest for self-awareness; this is not saying much, as the difference between the highest and the lowest positive sentiment was $6.08 \%$ (highest $=94.61 \%$; lowest $=88.53 \%$ ). Negative and neutral sentiments were very low-almost marginal-when it came to their positive sentiment counterparts, suggesting that when people were talking about emotional intelligence and the topics related to it, the driving factors were positivity in their text.

The findings showed that most of the people using the topics for their discussions of emotional intelligence were using Twitter to share their dealings in their personal, professional, and daily lives. These findings are consistent with previous studies which pertain to other fields (Stier et al. 2018; Wu et al. 2011; Kumar and Devi 2020). These were followed by EI advertisements, events, reports, and studies. As at the time of the study, the world was going through the distressful time of the pandemic, the platform of Twitter was being used as a tool to generate awareness about the advantages of being emotionally intelligent, which would explain the overwhelming positivity in the sentiments of the tweets, and the sparse but high user connectivity in the network analysis. Our findings on pandemicrelated emotional discourse are partially aligned with similar studies (Rufai and Bunce 2020; Xue et al. 2020; Arora et al. 2021). This may also explain the disproportionately high diffusion of topics of motivation, empathy, self-awareness, and emotional intelligence, even though other prominent factors contribute to the understanding of emotional intelligence.

Analyzing these discussions on Twitter provides new avenues in understanding the affective undertones of the users talking about a soft skill such as emotional intelligence. This study also shows that studying social media adds an invaluable source of information for professionals or academic researchers, and this information can be used to make more informed decisions and effectively deal with the issues attached to it. 


\section{Study Implications}

Twitter has been used by professionals in a myriad of ways (Vis 2013) and the findings of this study indicated that professionals having conversations on emotional intelligence are more conversational and information-focused than ideological. This is important for people pursuing careers in the domain of emotional intelligence because the business environment is dynamic and ever-changing, and it is adamant for these professionals to continually upgrade themselves with changes in the sources of knowledge on emotional intelligence.

It is also important to network and promote one's skill set and expertise on online platforms (Holmberg and Thelwall 2014; Conway et al. 2013; Nason et al. 2015), not only for business professionals but also academic researchers. Professionals can take advantage of these metrics for learning, networking, and promotion. Sentiment analysis and topic modeling can provide a general consensus of the important topics that are being discussed, and what the overall sentiment of these discussions is. Similarly, network analysis can show the communication patterns amongst the users of interest and measure the intensity of these communications.

This can also be used to understand the type of people one is about to hire in an organizations. Because organizations are swiftly becoming global and competitive, social media could become one of the better tools for hiring professionals who are not only experts in their domain of expertise, but are also socially adept enough to be intelligent in their adaptive behavior. Mining of user information can allow for a better perspective on these potential candidates through their tweets, retweets, followers, or other engagements.

Some important research implications can also be derived from this study. The amount of data shared under each of the main hashtags of each topic was astonishing over the month-long study. They were also significantly diverse, as seen in the network topology. Emotional intelligence is also very relevant to other areas of research, such as behavior, personality, leadership, mental health, etc. This is also consistent with the findings of empirical studies in the area (Pathak et al. 2018; Shankar and Tewari 2021; Shearer 2018). The interdisciplinary nature of EI opens up new sets of diversions and cohesion for the future.

\section{Limitations and Future Research}

Professionals and academic researchers in the area of emotional intelligence must use Twitter as an important source of information. However, the EI world has been tediously slow in figuring out the importance of effectively using social media and the prominence it plays in developing research, academia, and the industry. One limitation of this study is the data collection. The timeframe of collecting the data was very small-less than a month - and thus, a longer time duration would allow for a better understanding of the contextual nature of the conversations regarding emotional intelligence. Another thing was the abundance of the words and hashtags of emotional intelligence. Further studies can look into multiple words or hashtags, preferably the topics identified in this study, which would further provide a more comprehensive picture of the domain of study. Another way could be to use the preferential keywords that could be identified using analytical techniques to mine tweets that cover other factors influencing emotional intelligence that have been identified by other prominent researchers (Goleman 1995; Mayer et al. 2000).

It is important to build an understanding of how social media plays a prominent role in emotional intelligence. This has to be built through academic investigations and practical applications. This can be achieved by creating guidelines that allow an understanding of the diverse practices of emotional intelligence and innovation in academia, and also through measuring investments into the performance of emotionally intelligent individuals and teams. There is an increasing need in the area of emotional intelligence, and big data and social media can provide an incentivized breakthrough. 


\section{Conclusions}

In the present scenario of the dynamic environment, the study of emotional intelligence has increasingly become a significantly key research topic. The study of social media, big data, and natural language processing allows for analyzing, processing, and summarizing the information by using the affective and emotional spectrum of the content, which, in turn, allows measurement and analysis of the emotional competency of the users of online media. In the current domain of analysis of microblogging on emotional intelligence, there is an increasing need and demand for analyzing the role that emotional intelligence plays in users' interactions. This study also analyzed the hidden features and characteristics of the users and their shared content, and opens avenues for establishing the connections between the users and their shared content.

This study aimed to explore the issues and evaluate the role that emotional intelligence plays in the users' interactions on the microblogging giant Twitter, by analyzing the content through a framework that studies the descriptive, content, and network analytics (DA, CA, and NA). However, due to the limitations and other objective factors, there are still some areas that can be improved in future studies. For future work, researchers could conduct a more holistic emotional expression analysis that could comprehensively study the individual users' characteristics, behavior, and personality, and how these can affect their judgments and decision-making.

This is especially imperative for business organizations, as they are amongst the top contributors to the content on emotional intelligence on Twitter, and are amongst the most active and visible handles, which makes it clear that they are increasingly trying to use the platform and the theory of emotional intelligence to further better their business policies. This study thus opens up an avenue that can help both research academicians and industry professionals to increase their engagement and attention to further improve their workplace performance and outcomes.

Author Contributions: Conceptualization, S.S.; methodology, S.S.; software, S.S..; validation, V.T., and S.S.; formal analysis, S.S. and V.T.; investigation, S.S.; resources, S.S.; data curation, S.S.; writingoriginal draft preparation, S.S.; writing — review and editing, V.T. and S.S.; visualization, V.T.; supervision, V.T.; project administration, S.S. All authors have read and agreed to the published version of the manuscript.

Funding: This research received no external funding.

Institutional Review Board Statement: Not applicable.

Informed Consent Statement: The Data Extraction was done with the help of Twitter API after getting informed consent from the Twitter Developers.

Data Availability Statement: All the extracted data and supplemental materials can be found on the corresponding author's Github page at https:/ / github.com/theshane007/EITwitterData.

Acknowledgments: The authors would like to acknowledge Shashwat Shankar's invaluable help to this study, without whom this research could not be conducted.

Conflicts of Interest: The authors declare no conflict of interest.

\section{References}

Abkenar, Sepideh Bazzaz, Mostafa Haghi Kashani, Ebrahim Mahdipour, and Seyed Mahdi Jameii. 2020. Big data analytics meets social media: A systematic review of techniques, open issues, and future directions. Telematics and Informatics 57: 101517. [CrossRef]

Aiello, Luca Maria, Georgios Petkos, Carlos Martin, David Corney, Symeon Papadopoulos, Ryan Skraba, Ayse Göker, Ioannis Kompatsiaris, and Alejandro Jaimes. 2013. Sensing trending topics in Twitter. IEEE Transactions on Multimedia 15: 1268-82. [CrossRef]

Alotaibi, Shoayee, Rashid Mehmood, Iyad Katib, Omer Rana, and Aiiad Albeshri. 2020. Sehaa: A big data analytics tool for healthcare symptoms and diseases detection using Twitter, Apache Spark, and Machine Learning. Applied Sciences 10: 1398. [CrossRef]

Angiani, Giulio, Laura Ferrari, Tomaso Fontanini, Paolo Fornacciari, Eleonora Iotti, Federico Magliani, and Stefano Manicardi. 2016. A Comparison between Preprocessing Techniques for Sentiment Analysis in Twitter. Paper presented at the KDWeb, Cagliari, Italy, September 8-10. 
Arora, Anshika, Pinaki Chakraborty, M. P. S. Bhatia, and Prabhat Mittal. 2021. Role of emotion in excessive use of Twitter during COVID-19 imposed lockdown in India. Journal of Technology in Behavioral Science 6: 370-77. [CrossRef] [PubMed]

Bastian, Mathieu, Sebastien Heymann, and Mathieu Jacomy. 2009. Gephi: An open source software for exploring and manipulating networks. Paper presented at the International AAAI Conference on Web and Social Media, San Jose, CA, USA, May 17-20; vol. 3.

Binali, Haji, Chen Wu, and Vidyasagar Potdar. 2010. Computational approaches for emotion detection in text. Paper presented at the 4th IEEE International Conference on Digital Ecosystems and Technologies, Dubai, United Arab Emirates, April 12-15; pp. $172-77$.

Bogen, Katherine W., Kaitlyn K. Bleiweiss, Nykia R. Leach, and Lindsay M. Orchowski. 2020. Sexual victimization among men: A qualitative analysis of the twitter hashtag\# UsToo. Journal of Interpersonal Violence. [CrossRef]

Bollen, Johan, Huina Mao, and Xiaojun Zeng. 2011. Twitter mood predicts the stock market. Journal of Computational Science 2: 1-8. [CrossRef]

Bougie, Gargi, Jamie Starke, Margaret-Anne Storey, and Daniel M. German. 2011. Towards understanding twitter use in software engineering: Preliminary findings, ongoing challenges and future questions. Paper presented at the 2nd International Workshop on Web 2.0 for Software Engineering, Honolulu, HI, USA, May 24; pp. 31-36.

Bruns, Axel, and Jean Burgess. 2011. New methodologies for researching news discussion on Twitter. Paper presented at the 3rd Future of Journalism Conference 2011, Cardiff, UK, September 8-9; pp. 1-11.

Bryan, Victoria M., and John D. Mayer. 2021. Are People-Centered Intelligences Psychometrically Distinct from Thing-Centered Intelligences? A Meta-Analysis. Journal of Intelligence 9: 48. [CrossRef]

Burrus, Jeremy, Anthony Betancourt, Steven Holtzman, Jennifer Minsky, Carolyn MacCann, and Richard D. Roberts. 2012. Emotional intelligence relates to well-being: Evidence from the Situational Judgment Test of Emotional Management. Applied Psychology: Health and Well-Being 4: 151-66. [CrossRef] [PubMed]

Cao, Juan, Tian Xia, Jintao Li, Yongdong Zhang, and Sheng Tang. 2009. A density-based method for adaptive LDA model selection. Neurocomputing 72: 1775-81. [CrossRef]

Castillo, Carlos, Marcelo Mendoza, and Barbara Poblete. 2011. Information credibility on twitter. Paper presented at the 20th International Conference on World Wide Web, Hyderabad, India, March 28-April 1; pp. 675-84.

Cecere, Lora. 2012. Big Data: Go Big or Go Home. Philadelphia: Supply Chain Insights LLC.

Ceron, Andrea, Luigi Curini, and Stefano Maria Iacus. 2016. Politics and Big Data: Nowcasting and Forecasting Elections with Social Media. New York: Taylor \& Francis.

Chae, Bongsug Kevin. 2015. Insights from hashtag\# supplychain and Twitter Analytics: Considering Twitter and Twitter data for supply chain practice and research. International Journal of Production Economics 165: 247-59.

Chen, Aihui, Yaobin Lu, and Bin Wang. 2017. Customers' purchase decision-making process in social commerce: A social learning perspective. International Journal of Information Management 37: 627-38. [CrossRef]

Cinelli, Matteo, Walter Quattrociocchi, Alessandro Galeazzi, Carlo Michele Valensise, Emanuele Brugnoli, Ana Lucia Schmidt, Paola Zola, Fabiana Zollo, and Antonio Scala. 2020. The covid-19 social media infodemic. Scientific Reports 10: 1-10. [CrossRef] [PubMed]

Conway, Bethany Anne, Kate Kenski, and Di Wang. 2013. Twitter use by presidential primary candidates during the 2012 campaign. American Behavioral Scientist 57: 1596-610. [CrossRef]

Courtney, Karen L., Omid Shabestari, and Alex Mu-Hsing Kuo. 2013. The use of social media in healthcare: Organizational, clinical, and patient perspectives. In Enabling Health and Healthcare through ICT: Available, Tailored and Closer. Ancona: IOS Press Ebooks, vol. 183, p. 244.

Dale, Katherine R., Arthur A. Raney, Qihao Ji, Sophie H. Janicke-Bowles, Joshua Baldwin, Jerrica T. Rowlett, Cen Wang, and Mary Beth Oliver. 2020. Self-transcendent emotions and social media: Exploring the content and consumers of inspirational Facebook posts. New Media \& Society 22: 507-27.

Dodds, Peter Sheridan, Kameron Decker Harris, Isabel M. Kloumann, Catherine A. Bliss, and Christopher M. Danforth. 2011. Temporal patterns of happiness and information in a global social network: Hedonometrics and Twitter. PLoS ONE 6: e26752. [CrossRef] [PubMed]

Doldor, Elena, Madeleine Wyatt, and Jo Silvester. 2019. Statesmen or cheerleaders? Using topic modeling to examine gendered messages in narrative developmental feedback for leaders. The Leadership Quarterly 30: 101308. [CrossRef]

Ferrara, Emilio. 2020. Bots, elections, and social media: A brief overview. In Disinformation, Misinformation, and Fake News in Social Media. Cham: Springer, pp. 95-114.

Ford, Elizabeth, Keegan Curlewis, Akkapon Wongkoblap, and Vasa Curcin. 2019. Public opinions on using social media content to identify users with depression and target mental health care advertising: Mixed methods survey. JMIR Mental Health 6: e12942. [CrossRef] [PubMed]

Gao, Junling, Pinpin Zheng, Yingnan Jia, Hao Chen, Yimeng Mao, Suhong Chen, Yi Wang, Hua Fu, and Junming Dai. 2020. Mental health problems and social media exposure during COVID-19 outbreak. PLoS ONE 15: e0231924. [CrossRef] [PubMed]

Gokulakrishnan, Balakrishnan, Pavalanathan Priyanthan, Thiruchittampalam Ragavan, Nadarajah Prasath, and AShehan Perera. 2012. Opinion mining and sentiment analysis on a twitter data stream. Paper presented at the International Conference on Advances in ICT for Emerging Regions (ICTer2012), Colombo, Sri Lanka, December 12-15; pp. 182-88.

Goleman, Daniel. 1995. Emotional Intelligence: Why It Can Matter More Than IQ. New York: Bantam Books. 
Gorodnichenko, Yuriy, Tho Pham, and Oleksandr Talavera. 2018. Social Media, Sentiment and Public Opinions: Evidence from\# Brexit and\# USElection (No. w24631). Cambridge: National Bureau of Economic Research.

Hall, Judith A., and Marianne Schmid Mast. 2007. Sources of accuracy in the empathic accuracy paradigm. Emotion 7: 438. [CrossRef] [PubMed]

Hickerson, Andrea, and Ammina Kothari. 2017. Learning in public: Faculty and student opinions about social media in the classroom. Journalism \& Mass Communication Educator 72: 397-409.

Holmberg, Kim, and Mike Thelwall. 2014. Disciplinary differences in Twitter scholarly communication. Scientometrics 101: 1027-42. [CrossRef]

Hornung, Olivia, Sven Dittes, and Stefan Smolnik. 2018. When Emotions Go Social-Understanding The Role Of Emotional Intelligence In Social Network USE. Paper presented at the Twenty-Sixth European Conference on Information Systems (ECIS 2018), Portsmouth, UK, June 23-28. Research-in-Progress Papers.

Huang, Jia-Yen, and Jin-Hao Liu. 2020. Using social media mining technology to improve stock price forecast accuracy. Journal of Forecasting 39: 104-16. [CrossRef]

İş, Hafzullah, and Taner Tuncer. 2018. Confidence index analysis of Twitter users timeline. Paper presented at the 2018 International Conference on Artificial Intelligence and Data Processing (IDAP), Malatya, Turkey, September 28-30; pp. 1-8.

Israelashvili, Jacob, Lisanne S. Pauw, Disa A. Sauter, and Agneta H. Fischer. 2021. Emotion Recognition from Realistic Dynamic Emotional Expressions Cohere with Established Emotion Recognition Tests: A Proof-of-Concept Validation of the Emotional Accuracy Test. Journal of Intelligence 9: 25. [CrossRef] [PubMed]

Jaidka, Kokil, Saifuddin Ahmed, Marko Skoric, and Martin Hilbert. 2019. Predicting elections from social media: A three-country, three-method comparative study. Asian Journal of Communication 29: 252-73. [CrossRef]

Jain, Vinay Kumar, Shishir Kumar, and Steven Lawrence Fernandes. 2017. Extraction of emotions from multilingual text using intelligent text processing and computational linguistics. Journal of Computational Science 21: 316-26. [CrossRef]

Jin, Seung-A. Annie. 2012. The potential of social media for luxury brand management. Marketing Intelligence E Planning 30: 687-99.

Jurgens, David, Tyler Finethy, James McCorriston, Yi Tian Xu, and Derek Ruths. 2015. Geolocation prediction in twitter using social networks: A critical analysis and review of current practice. Paper presented at the International AAAI Conference on Web and Social Media, Oxford, UK, May 26-29; vol. 9.

Kadam, Abhay B., and Sachin R. Atre. 2020. Negative impact of social media panic during the COVID-19 outbreak in India. Journal of Travel Medicine 27: taaa057. [CrossRef] [PubMed]

Kersten, Jens, and Friederike Klan. 2020. What happens where during disasters? A Workflow for the multifaceted characterization of crisis events based on Twitter data. Journal of Contingencies and Crisis Management 28: 262-80. [CrossRef]

Kosinski, Michal, Yoram Bachrach, Pushmeet Kohli, David Stillwell, and Thore Graepel. 2014. Manifestations of user personality in website choice and behaviour on online social networks. Machine Learning 95: 357-80. [CrossRef]

Kumar, R. Kiran, and P. Bharathi Devi. 2020. Emotional Intelligence of People Towards Political Parties in Andhra Pradesh Based on Twitter Data. In ICDSMLA 2019. Singapore: Springer, pp. 626-36.

Kushwaha, Amit Kumar, Arpan Kumar Kar, and P. Vigneswara Ilavarasan. 2020. Predicting information diffusion on twitter a deep learning neural network model using custom weighted word features. Paper presented at the 19th IFIP WG 6.11 Conference on e-Business, e-Services, and e-Society, I3E 2020, Skukuza, South Africa, April 6-8. Cham: Springer, pp. 456-68.

Lalicic, Lidija, Assumpció Huertas, Antonio Moreno, and Mohammed Jabreel. 2020. Emotional brand communication on Facebook and Twitter: Are DMOs successful? Journal of Destination Marketing \& Management 16: 100350.

Lim, Ming D., and Damian P. Birney. 2021. Experiential and Strategic Emotional Intelligence Are Implicated When Inhibiting Affective and Non-Affective Distractors: Findings from Three Emotional Flanker N-Back Tasks. Journal of Intelligence 9: 12. [CrossRef] [PubMed]

Madaan, Rosy, Komal Kumar Bhatia, and Surbhi Bhatia. 2020. Understanding the role of emotional intelligence in usage of social media. Paper presented at the 2020 10th International Conference on Cloud Computing, Data Science \& Engineering (Confluence), Noida, India, January 29-31; pp. 586-91.

Malhotra, Arvind, Claudia Kubowicz Malhotra, and Alan See. 2012. How to get your messages retweeted. MIT Sloan Management Review 53: 61-66.

Mann, Jordan. 2014. Social Media offers opportunities for Supply Chain Management-Social Media for Business Performance. Available online: https://smbp.uwaterloo.ca/2014/07/social-media-offers-opportunities-for-supply-chain-management/ (accessed on 23 March 2021).

Mao, Huina, Scott Counts, and Johan Bollen. 2011. Predicting financial markets: Comparing survey, news, twitter and search engine data. arXiv arXiv:1112.1051.

Masip, Pere, Carlos Ruiz-Caballero, Jaume Suau, and David Puertas. 2020. Participation and MediaMedia and Twitter Agendas for Social Mobilizations: The Case of the Protests in Defense of the Public Healthcare System in Spain. International Journal of Communication 14: 22.

Mayer, John D., Peter Salovey, David R. Caruso, and Robert Jeffrey Sternberg. 2000. Models of emotional intelligence. In Emotional Intelligence: Key Readings on the Mayer and SALOVEY Model. Katonah: National Professional Resources, Inc., pp. 81-119. 
Menon, Vishnu M., and H. A. Rahulnath. 2016. A novel approach to evaluate and rank candidates in a recruitment process by estimating emotional intelligence through social media data. Paper presented at the 2016 International Conference on Next Generation Intelligent Systems (ICNGIS), Kottayam, India, September 1-3; pp. 1-6.

Moeller, Julia, Zorana Ivcevic, Marc A. Brackett, and Arielle E. White. 2018. Mixed emotions: Network analyses of intra-individual co-occurrences within and across situations. Emotion 18: 1106. [CrossRef] [PubMed]

Nason, Gregory J., Fardod O'Kelly, Michael E. Kelly, Nigel Phelan, Rustom P. Manecksha, Nathan Lawrentschuk, and Declan G. Murphy. 2015. The emerging use of T witter by urological journals. BJU International 115: 486-90. [CrossRef]

Natoli, Paula. 2013. The Impact of Social Media on the Supply Chain: Is There One? Available online: https://blog.blueyonder.com/ the-impact-of-social-media-on-the-supply-chain-is-there-one/ (accessed on 23 March 2021).

O'leary, Daniel E. 2011. The use of social media in the supply chain: Survey and extensions. Intelligent Systems in Accounting, Finance and Management 18: 121-44. [CrossRef]

Pathak, Arjit, Vijayshri Tewari, and Shardul Shankar. 2018. Impact of Emotional Intelligence on employability of IT professionals. Management Insight 14: 14-21. [CrossRef]

Paul, Newly, and Mingxiao Sui. 2019. I Can Feel What You Feel: Emotion Exchanges in Twitter Conversations between Candidates and the Public. Journal of Political Marketing. [CrossRef]

Pizzuti, Adam G., Karan H. Patel, Erin K. McCreary, Emily Heil, Christopher M. Bland, Eric Chinaeke, Bryan L. Love, and P. Brandon Bookstaver. 2020. Healthcare practitioners' views of social media as an educational resource. PLoS ONE 15: e0228372. [CrossRef] [PubMed]

Rufai, Sohaib R., and Catey Bunce. 2020. World leaders' usage of Twitter in response to the COVID-19 pandemic: A content analysis. Journal of Public Health 42: 510-16. [CrossRef]

Ruz, Gonzalo A., Pablo A. Henríquez, and Aldo Mascareño. 2020. Sentiment analysis of Twitter data during critical events through Bayesian networks classifiers. Future Generation Computer Systems 106: 92-104. [CrossRef]

Sailunaz, Kashfia, and Reda Alhajj. 2019. Emotion and sentiment analysis from Twitter text. Journal of Computational Science 36 : 101003. [CrossRef]

Shankar, Shardul, and Vijayshri Tewari. 2021. Impact of Collective Intelligence and Collective Emotional Intelligence on the Psychological Safety of the Organizations. Vision. [CrossRef]

Sharma, Megha, Kapil Yadav, Nitika Yadav, and Keith C. Ferdinand. 2017. Zika virus pandemic-Analysis of Facebook as a social media health information platform. American Journal of Infection Control 45: 301-2. [CrossRef] [PubMed]

Shearer, Branton. 2018. Multiple intelligences in teaching and education: Lessons learned from neuroscience. Journal of Intelligence 6: 38. [CrossRef]

So, Kevin Kam Fung, Laurie Wu, Lina Xiong, and Ceridwyn King. 2018. Brand management in the era of social media: Social visibility of consumption and customer brand identification. Journal of Travel Research 57: 727-42. [CrossRef]

Sóti, Attila, Alessandro Ancarani, József Stéger, and Gábor Vattay. 2020. Influence of Twitter activity on the stock price of soccer clubs. Social Network Analysis and Mining 10: 1-12. [CrossRef]

Souza, Thársis Tuani Pinto, Olga Kolchyna, Philip C. Treleaven, and Tomaso Aste. 2015. Twitter sentiment analysis applied to finance: A case study in the retail industry. arXiv arXiv:1507.00784.

Stieglitz, Stefan, and Linh Dang-Xuan. 2013. Emotions and information diffusion in social media-Sentiment of microblogs and sharing behavior. Journal of Management Information Systems 29: 217-48. [CrossRef]

Stier, Sebastian, Arnim Bleier, Haiko Lietz, and Markus Strohmaier. 2018. Election campaigning on social media: Politicians, audiences, and the mediation of political communication on Facebook and Twitter. Political Communication 35: 50-74. [CrossRef]

Suhasini, Matla, and Badugu Srinivasu. 2020. Emotion detection framework for twitter data using supervised classifiers. In Data Engineering and Communication Technology. Singapore: Springer, pp. 565-76.

Tago, Kiichi, and Qun Jin. 2018. Influence analysis of emotional behaviors and user relationships based on Twitter data. Tsinghua Science and Technology 23: 104-13. [CrossRef]

Talbot, Catherine V., Siobhan T. O'Dwyer, Linda Clare, and Janet Heaton. 2021. The use of Twitter by people with young-onset dementia: A qualitative analysis of narratives and identity formation in the age of social media. Dementia. [CrossRef] [PubMed]

Tankovska, H. 2021. Twitter: Monthly Active Users Worldwide IStatista. Statista. Available online: https://www.statista.com/ statistics/282087/number-of-monthly-active-twitter-users/?Tag=Inbound\%202012 (accessed on 23 March 2021).

Tellez, Eric S., Sabino Miranda-Jiménez, Mario Graff, Daniela Moctezuma, Oscar S. Siordia, and Elio A. Villaseñor. 2017. A case study of Spanish text transformations for twitter sentiment analysis. Expert Systems with Applications 81: 457-71. [CrossRef]

Terry, Mark. 2009. Twittering healthcare: Social media and medicine. Telemedicine and e-Health 15: 507-10. [CrossRef]

Thelwall, Mike, Kevan Buckley, and Georgios Paltoglou. 2011. Sentiment in Twitter events. Journal of the American Society for Information Science and Technology 62: 406-18. [CrossRef]

Tsui, Derek. 2017. Predicting Stock Price Movement Using Social Media Analysis. Technical Report. Stanford: Stanford University.

Twitter. 2020. Available online: https:/ /investor.twitterinc.com/home/default.aspx (accessed on 23 March 2021).

Twitter. 2021. About Twitter I Our Company and Priorities. Available online: https:/ /about.twitter.com/ (accessed on 23 March 2021).

Vega, Edgardo, Ramanujam Parthasarathy, and Josette Torres. 2010. Where Are My Tweeps?: Twitter Usage at Conferences. Available online: https:/ / www.slideshare.net/ramanuj/twitter-usage-at-conferences (accessed on 23 March 2021). 
Vis, Farida. 2013. Twitter as a reporting tool for breaking news: Journalists tweeting the 2011 UK riots. Digital Journalism 1: 27-47. [CrossRef]

Wang, Yichen, and Aditya Pal. 2015. Detecting emotions in social media: A constrained optimization approach. Paper presented at the Twenty-Fourth International Joint Conference on Artificial Intelligence, Buenos Aires, Argentina, July 25-31.

Wang, Yan, Haiyan Hao, and Lisa Sundahl Platt. 2021. Examining risk and crisis communications of government agencies and stakeholders during early-stages of COVID-19 on Twitter. Computers in Human Behavior 114: 106568. [CrossRef]

Watts, Duncan J. 2004. Six Degrees: The Science of a Connected Age. New York: WW Norton \& Company.

Wei, Kai, Daniel Jacobson López, and Shiyou Wu. 2019. The Role of Language in Anti-Immigrant Prejudice: What Can We Learn from Immigrants' Historical Experiences? Social Sciences 8: 93. [CrossRef]

$\mathrm{Wu}$, Shaomei, Jake M. Hofman, Winter A. Mason, and Duncan J. Watts. 2011. Who says what to whom on twitter. Paper presented at the 20th International Conference on World Wide Web, Hyderabad, India, March 28; pp. 705-14.

Xu, Guandong, Yanchun Zhang, and Xun Yi. 2008. Modelling user behaviour for web recommendation using lda model. Paper presented at the 2008 IEEE/WIC/ACM International Conference on Web Intelligence and Intelligent Agent Technology, Sydney, NSW, Australia, December 9-12; vol. 3, pp. 529-32.

Xue, Jia, Junxiang Chen, Ran Hu, Chen Chen, Chengda Zheng, Yue Su, and Tingshao Zhu. 2020. Twitter discussions and emotions about the COVID-19 pandemic: Machine learning approach. Journal of Medical Internet Research 22: e20550. [CrossRef] [PubMed]

Zafar, Abaid Ullah, Jiangnan Qiu, Mohsin Shahzad, Jie Shen, Tahseen Ahmed Bhutto, and Muhammad Irfan. 2020. Impulse buying in social commerce: Bundle offer, top reviews, and emotional intelligence. Asia Pacific Journal of Marketing and Logistics 33: 945-73. [CrossRef]

Zeng, Daniel, Hsinchun Chen, Robert Lusch, and Shu-Hsing Li. 2010. Social media analytics and intelligence. IEEE Intelligent Systems 25: 13-16. [CrossRef]

Zhang, Ling, Magie Hall, and Dhundy Bastola. 2018. Utilizing Twitter data for analysis of chemotherapy. International Journal of Medical Informatics 120: 92-100. [CrossRef] [PubMed]

Zhang, Yazhou, Dawei Song, Peng Zhang, Xiang Li, and Panpan Wang. 2019. A quantum-inspired sentiment representation model for twitter sentiment analysis. Applied Intelligence 49: 3093-108. [CrossRef]

Zhao, Shi, Qianyin Lin, Jinjun Ran, Salihu S. Musa, Guangpu Yang, Weiming Wang, Yijun Lou, Daozhou Gao, Lin Yang, Daihai He, and et al. 2020. Preliminary estimation of the basic reproduction number of novel coronavirus (2019-nCoV) in China, from 2019 to 2020: A data-driven analysis in the early phase of the outbreak. International Journal of Infectious Diseases 92: 214-17. [CrossRef] [PubMed]

Zimbra, David, Manoochehr Ghiassi, and Sean Lee. 2016. Brand-related Twitter sentiment analysis using feature engineering and the dynamic architecture for artificial neural networks. Paper presented at the 201649 th Hawaii International Conference on System Sciences (HICSS), Koloa, HI, USA, January 5-8; pp. 1930-38. 